\title{
475667 - BLOOD TRANSFUSION PREDICTORS IN LIVER RESECTION SURGERY
}

\author{
Nasr Hegazy, MD, Gordon Tait, PhD, Coimbatore Srinivas, MD, Stuart \\ McCLuskey, MD, PhD \\ Anesthesia, Toronto General Hospital, Toronto, ON, Canada
}

Introduction: One of the major issues of liver resections is blood loss necessitating perioperative blood transfusion. Blood transfusion is independently associated with serious postoperative adverse events. The objective of this study was to identify perioperative predictors of blood transfusion in patients undergoing liver resections. Predicting which patients are at high risk of blood loss requiring transfusion will allow us to introduce targeted interventions to reduce this complication.

Methods: Following institutional approval, data on all patients undergoing liver resections at the an academic institution between January , 2004 and December , 2006 were collected from the patients chart retrospectively. Perioperaive variables extracted include: demographics, primary hepatic disease, comorbidities (diabetes mellitus, systemic hypertension, renal insufficiency/failure), smoking history, medications (antiplatelet and anticoagulants), laboratory parameters (hemoglobin concentration (Hb), platelet count, PTT/INR), surgical variables (type of anesthesia, duration of the surgery and intraoperative fluid management). Statistical analyses were performed using SAS version 9.1 (SAS institute, Cary, NC). Unadjusted association of potential predictors of transfusion was evaluated for both continuous (t-test) and categorical (Chi-squared test) variables. Independent predictors of transfusion were identified by multivariable logistic regression analysis.

Results: 66 out of 300 patients (22\%) received intraoperative blood transfusion. Unadjusted univariate analyses identified 8 predictors of RBC transfusion (Age, weight, height, pre op $\mathrm{Hb}$, pre op INR, platelet count, intraoperative crystalloid volume and use of Pentaspan $>500 \mathrm{ml}$ ). In multivariable logistic regression 5 independent predictors were identified. The model is well calibrated (Hosmer-Lemeshow test $\mathrm{P}=0.592$ ) and is highly discriminative (ROC area 0.842).

Discussion: RBC transfusion in liver resections can be reliably predicted by readily available pre and intraoperative variables. While this model requires validation, it may be used to improve the perioperative management of patient undergoing liver resection and their clinical outcome.

References: None 


\begin{tabular}{|c|c|c|c|}
\hline Variable & Odds Ratio & 95\% Confidence Interval & p value \\
\hline Pentaspan $>500 \mathrm{ml}$ & 5.77 & $2.890-11.513$ & 0.0001 \\
\hline Age & 1.031 & $1.009-1.053$ & 0.0051 \\
\hline Height & 0.984 & $0.973-0.995$ & 0.0048 \\
\hline Preoperative HB & 0.948 & $0.927-0.969$ & 0.0001 \\
\hline Crystalloid & 1.566 & $1.295-1.894$ & 0.0001 \\
\hline
\end{tabular}

